\title{
Genome-wide identification, characterisation and functional evaluation of WRKY genes in the sweet potato wild ancestor Ipomoea trifida (H.B.K.) G. Don. under abiotic stresses
}

\author{
Yuxia $\mathrm{Li}^{1+}$, Lei Zhang ${ }^{1+}$, Panpan Zhu ${ }^{2+}$, Qinghe $\mathrm{CaO}^{3}$, Jian Sun ${ }^{1}$, Zongyun $\mathrm{Li}^{1 *}$ and Tao Xu ${ }^{1 *}$ (D)
}

\begin{abstract}
Background: WRKY DNA-binding protein (WRKY) is a large gene family involved in plant responses and adaptation to salt, drought, cold and heat stresses. Sweet potato from the genus Ipomoea is a staple food crop, but the WRKY genes in Ipomoea species remain unknown to date. Hence, we carried out a genome-wide analysis of WRKYs in Ipomoea trifida (H.B.K.) G. Don., the wild ancestor of sweet potato.

Results: A total of 83 WRKY genes encoding 96 proteins were identified in I. trifida, and their gene distribution, duplication, structure, phylogeny and expression patterns were studied. ItfWRKYS were distributed on 15 chromosomes of I. trifida. Gene duplication analysis showed that segmental duplication played an important role in the WRKY gene family expansion in I. trifida. Gene structure analysis showed that the intron-exon model of the ItfWRKY gene was highly conserved. Meanwhile, the ItfWRKYs were divided into five groups (I, $\|\mathrm{a}+\| \mathrm{lb},\|\mathrm{c},\| \mathrm{d}+\| \mathrm{le}$ and III) on the basis of the phylogenetic analysis on I. trifida and Arabidopsis thaliana WRKY proteins. In addition, gene expression profiles confirmed by quantitative polymerase chain reaction showed that ItfWRKYS were highly up-regulated or down-regulated under salt, drought, cold and heat stress conditions, implying that these genes play important roles in response and adaptation to abiotic stresses.
\end{abstract}

Conclusions: In summary, genome-wide identification, gene structure, phylogeny and expression analysis of WRKY gene in 1. trifida provide basic information for further functional studies of ItfWRKYs and for the molecular breeding of sweet potato.

Keywords: WRKY, Transcription factor, Sweet potato, Ipomoea trifida, Abiotic stress

\section{Background}

Due to immobility, plants often encounter challenges from a number of abiotic environments [1]. To adapt to various stress conditions, plants have evolved a series of strategies at multiple levels. At the transcription level, regulating and inducing the temporal and spatial expression of transcription factor (TF) genes are the important approaches to obtain plant stress resistance [2].

\footnotetext{
* Correspondence: zongyunli@jsnu.edu.cn; xutao_yr@126.com

†Yuxia Li, Lei Zhang and Panpan Zhu contributed equally to this work.

${ }^{1}$ Key lab of phylogeny and comparative genomics of the Jiangsu province, Institute of Integrative Plant Biology, School of Life Sciences, Jiangsu Normal University, Xuzhou 221116, Jiangsu Province, China

Full list of author information is available at the end of the article
}

The WRKY protein contains a highly conserved 60 amino acid-long WRKY domain at the $\mathrm{N}$-termini and a zinc-finger-like motif $\left(\mathrm{CX}_{4-5} \mathrm{CX}_{22-23} \mathrm{HXH}\right)$ at the Ctermini [3-5]. WRKY TFs are involved not only in biotic stress responses $[3,6,7]$, seed coat and trichome development [8-10], embryogenesis [11] and leaf senescence $[12,13]$ but also in abiotic stress responses and adaptations. In A. thaliana, heat treatment inhibits the expression of AtWRKY33 while induces the expression of AtWRKY25 and AtWRKY26 [14]. VvWRKY24 is induced by cold treatment [15]. Pollen specific expressing gene AtWRKY34 negatively mediates the cold sensitivity of Arabidopsis pollen [16]. Overexpression of AtWRKY25, AtWRKY26, AtWRKY39 and TaWRKY33 can enhance

(c) The Author(s). 2019 Open Access This article is distributed under the terms of the Creative Commons Attribution 4.0 International License (http://creativecommons.org/licenses/by/4.0/), which permits unrestricted use, distribution, and reproduction in any medium, provided you give appropriate credit to the original author(s) and the source, provide a link to the Creative Commons license, and indicate if changes were made. The Creative Commons Public Domain Dedication waiver (http://creativecommons.org/publicdomain/zero/1.0/) applies to the data made available in this article, unless otherwise stated. 
plant resistance to heat stress $[14,17,18]$. Transgenic plants overexpressing WRKY genes show increased tolerance to salt and drought stresses, such as overexpressing rice gene OsWRKY45 and OsWRKY72 into rice [19, 20], wheat gene TaWRKY10 into tobacco [21], Brassica campestris gene BcWRKY46 and barley gene HvWRKY38 into A. thaliana $[22,23]$ and Gossypium hirsutum gene GhWRKY17 in Nicotiana [24]. Constitutive expression of corn gene ZmWR KY23 in Arabidopsis also increases the salt tolerance of plants [25]. The AtWRKY33 and AtWRKY25 double mutants are sensitive to $\mathrm{NaCl}$, and overexpression of any gene enhances the salt tolerance of $A$. thaliana [14]. In addition, overexpression of Dendranthema grandiflorum gene $D g W R$ $K Y 1$ or $D g W R K Y 3$ enhances the salt tolerance of tobacco [21]. Overexpression of GhWRKY25 increases the salt tolerance but reduces the drought tolerance of $A$. thaliana [26]. BhWRKY1 can bind BhGolS1 and regulate BhGolS1 under drought stress [27]. GmWRKY54 positively regulates the resistance to drought stress in A. thaliana [28]. Overexpression of GsWRKY20 reduces the stomatal density and water loss efficiency of $A$. thaliana, thus improving plant drought tolerance [29]. These findings suggest that WRKY TFs are potential targets for improving the abiotic stress resistance of crops. However, the WRKY genes in sweet potato remain largely unknown.

Sweet potato (Ipomoea batatas) of the family Convolvulaceae is a widely cultivated food crop with excellent agricultural traits. Meanwhile, it is a major forage crop and important bioenergy crop in China. However, the yield and quality of sweet potatoes have been reduced by various environmental pressures [30-35]. Diploid I. trifida, as the wild ancestor of cultivated sweet potato [36-38], contains many excellent characteristics, rendering it suitable good model species for the study of sweet potato breeding, construction of a transgenic system and self-incompatibility [39]. In the present study, we performed a genome-wide identification of I. trifida WRKY family members. Gene duplication, intron/exon distribution and the phylogenetic relationship were analysed. The tissue-specific and stress-responsive expression patterns of ItfWRKYs were observed. Moreover, the potential functions of ItfWRKYs were predicted and discussed. Our work provides basic information for further functional studies of $\operatorname{It} f W R K Y S$ and for the molecular breeding of sweet potato in the future.

\section{Results}

\section{Identification of ItfWRKYs}

Ninety-six WRKY proteins encoded by 83 WRKY genes were identified in I. trifida. These WRKYS are designated as ItfWRKY1-ItfWRKY83 according to their positions on the chromosome. Different transcripts from one gene are named similarly. For example, the three transcripts encoded by ItfWRKY43 are designated as ItfWRKY43.1, ItfWRKY43.2 and ItfWRKY43.3 (Additional file 1: Table
S1). As shown in Additional file 2: Fig. S1, all 96 ItfWRKY proteins contained one or two WRKY domains. Although the WRKYGQK domain is highly conserved, glutamine is replaced by a lysine residue in some WRKY proteins (e.g. ItfWRKY1, $-6,-18,-31,-32,-45$ and -76 ) (Additional file 2: Fig. S1), which are also found in WRKYs of tomato, Arabidopsis and other plant species [3, 40-42]. Similarly, two residues of ItfWRKY36 were replaced, in which glutamine was replaced by threonine residue and lysine was replaced by arginine residue. In addition, most of the ItfWRKY proteins contain C-X4-7-C-X23-H motifs to form $\mathrm{C} 2 \mathrm{H} 2 / \mathrm{C} 2 \mathrm{HC}$-type zinc finger structures (Additional file 2: Fig. S1).

Then, ItfWRKY protein size, protein molecular weight $(\mathrm{MW})$, isoelectric point ( $\mathrm{pI})$, phosphorylation site and subcellular location were analysed (Additional file 3: Table S2). Results showed that these 96 ItfWRKYs are 116 (ItfWRKY70) to 697 (ItfWRKY38) aa in length. The MW of the proteins is mostly between 20 and 50 kilodaltons $(\mathrm{kDa})$, and the pIs of these proteins are between 4.92 (ItfWRKY72) and 10.31 (ItfWRKY40). The pIs of about $60 \%$ proteins are lower than 7 , indicating that most ItfWRKY proteins are acidic under physiological conditions. However, the difference in PI of ItfWRKY proteins under physiological conditions leads to the difference in charge status of groups, causing the binding or dissociation of groups from the proteins and further affecting the protein function. As shown in Additional file 3: Table S2, ItfWRKYs contain 1-10 phosphorylation sites, of which ItfWRKY42, ItfWRKY55 and ItfWRKY75 have at most 10 phosphorylation sites. About $21 \%$ of ItfWRKYs contain six or more phosphorylation sites. However, some ItfWRKYs, such as ItfWRKY1, ItfWRKY6 and ItfWRKY31, do not have phosphorylation sites. All ItfWRKYs are predicted to be located in the chloroplast (Additional file 3: Table S2).

\section{Chromosomal locations and duplication events of the ItfWRKY gene family}

Among 83 ItfWRKY genes, 82 ItfWRKYs were mapped on 15 chromosomes, while one WRKY (ItfWRKY1) was located on the unanchored scaffold. Figure 1 shows two ItfWRKYs on chromosome (Chr) 8 and Chr 15; three on Chr 7 and Chr 14; four on Chr 9, Chr 11 and Chr 13; six on Chr 2 and Chr 4; seven on Chr 3, Chr 6 and Chr 12; eight on Chr 1; nine on Chr 10; and ten ItfWRKY genes on Chr 5, indicating that ItfWRKYS are distributed unevenly on chromosomes. Segmental and tandem duplication is a main approach for plant gene family expansion [43]. Segmental duplication duplicates multiple genes through polyploidy followed by chromosome rearrangements [43, 44], while tandem duplication is characterised as multiple members of one family occurring within the same intergenic region or in neighbouring intergenic regions (within $200 \mathrm{~kb}$ ) [45]. We conducted a collinear analysis to study the possible gene 


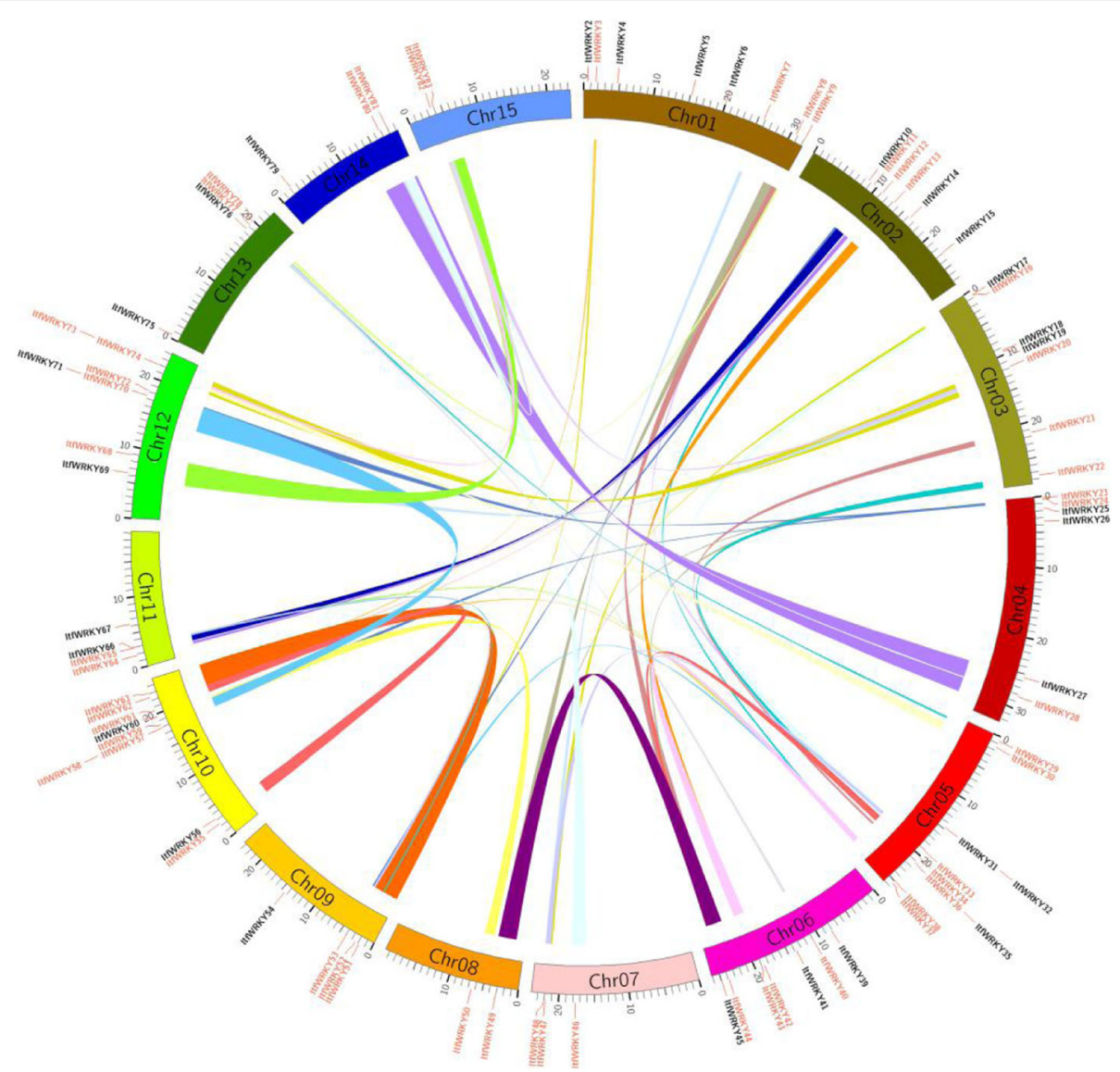

Fig. 1 Distribution and segmental duplication of ItfWRKYS in I. trifida chromosomes. Exactly 82 ItfWRKYs were mapped to 15 chromosomes, while one WRKY (ItfWRKY1) was located on the unanchored scaffold. Different coloured lines indicate segmental duplication. The red line next to the name indicates the gene cluster on each chromosome. Gene names with collinearity are coloured in red, and no collinear gene names are coloured in black

duplication types (Fig.1 and Additional file 4: Table S3). Fifty collinear fragments, including ItfWRKY3-ItfWRKY52, ItfWRKY3-ItfWRKY62, ItfWRKY7-ItfWRKY47, ItfWRKY8ItfWRKY34 and ItfWRKY12-ItfWRKY64, were found using protein-protein BLAST (BLASTP) and Multiple Collinearity Scan tool kit X version (MCSCANX). The above results indicate that segmental duplication played an important role in the ItfWRKY family expansion.

\section{Gene structure analysis of ItfWRKYs}

To identify the structural features of ItfWRKYS, we analysed the ItfWRKY gene structures. Results showed that the intron number of $I t f W R K Y$ gene family members ranges from 1 to 7, except ItfWRKY36, ItfWRKY50, ItfWRKY58 and ItfWRKYY76 without any intron. Among 96 transcripts, 45 (47\%) transcripts contain 2 introns, which occupied the largest percentage. Forty (42\%) transcripts contain 3-6 introns. ItfWRKY48 contains the most introns (7) in the ItfWRKY gene family. The diversity in the number of exons and introns leads to a variety of mRNA splicing results during mRNA post-processing, which may be related to protein diversity. As shown in Fig. 2, ItfWRKYs were classified into five groups (Cluster I, IIa + IIb, IIc, IId + IIe and III) on the basis of the topology of the Neighbour-joining (NJ) phylogenetic tree (Fig. 2).

\section{Phylogenetic analysis of ItfWRKY proteins}

To study the evolutionary relationship of WRKYs between I. trifida and A. thaliana, we established a phylogenetic tree of WRKYs. As shown in Fig. 3, the tree contains 72 AtWR KY and 96 ItfWRKY proteins. The A. thaliana AtWRKYs were divided into five subgroups (I, IIa + IIb, IIc, IId + IIe and III) [3, 21]. Similar to AtWRKYs, ItfWRKYs were also divided into five subgroups. The ItfWRKYs contain two WRKY domains in Group I, one WRKY domain (with the same Cys2-His2 zinc-finger motif) in Group II and one WRKY domain (with different Cys2-His/Cys Cys2-His2 zinc finger motifs) in Group III. Among these ItfWRKY proteins, 61 in Group II formed the largest branches, of which $5+13,25$ and $6+12$ ItfWRKY proteins were assigned to subgroups IIa + IIb, IIc and IId + IIe, respectively. The second largest group, Group I has 48 WRKYs, 


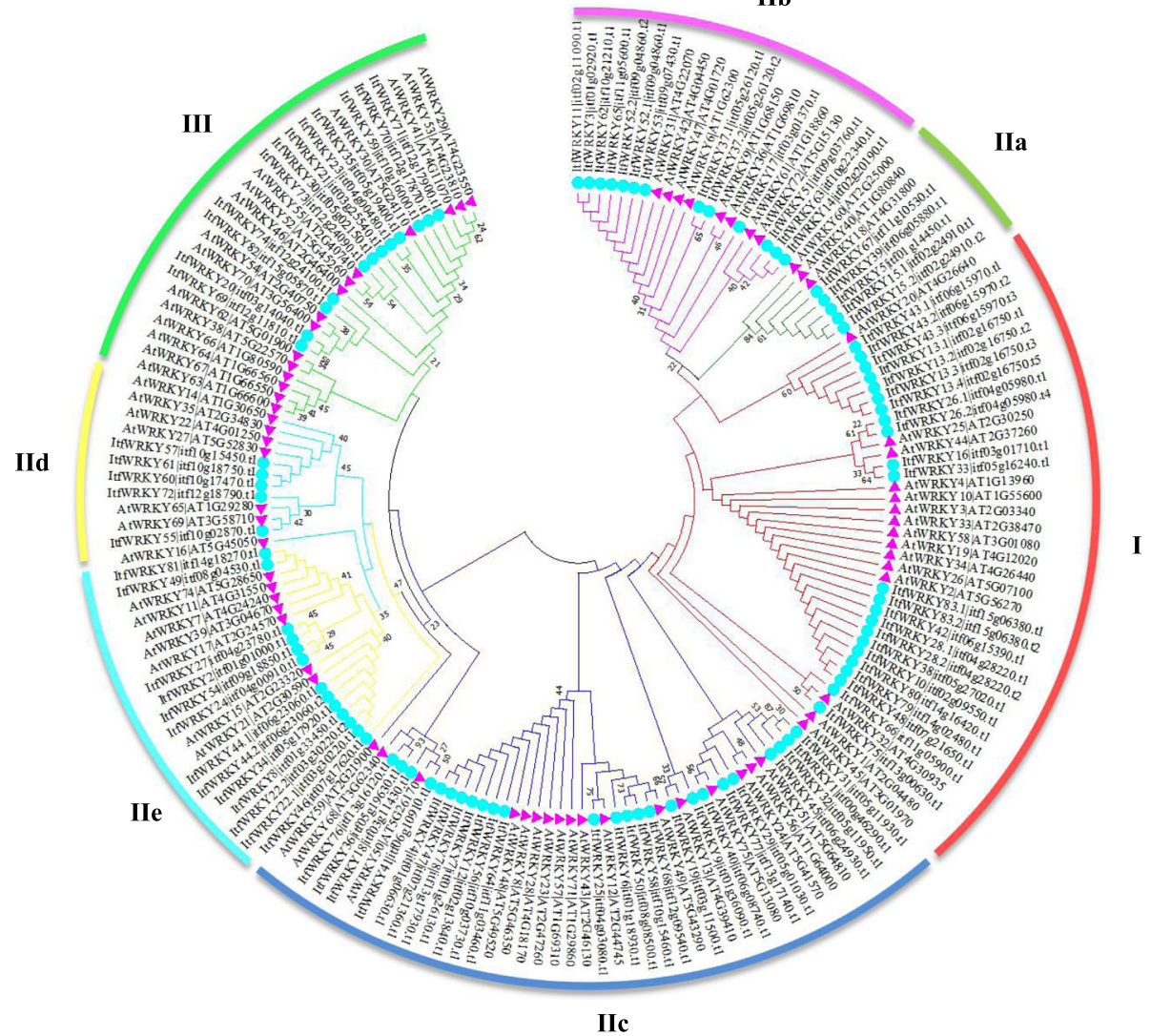

Fig. 2 Phylogenetic relationships among the identified WRKY proteins in Arabidopsis and I. trifida. The 72 Arabidopsis and 96 I. trifida WRKY protein sequences were used to construct the phylogenetic tree using MEGA7 by the Maximum Likelihood method analysis (1000 replicates). Arabidopsis and I. trifida genes were indicated at the end of the branches. Subgroups I, Ila, IIb, Ilc, IId, Ile and III were named according to Arabidopsis. The coloured regions indicate different subfamilies. The blue solid circles and the purple solid triangle indicate the ItfWRKY and AtWRKY proteins, respectively

including 23 ItfWRKYs and 15 AtWRKYs. Group III has only 27 members (12 ItfWRKYs and 15 AtWRKYs). In addition, the subfamily classification generated from WRKY proteins is consistent with the subgroup classification generated from the intron/exon gene structure analysis.

\section{Interaction network of the ItfWRKY proteins}

Understanding the functional relationship of ItfWRKYs is important to understand the regulatory pathway of the family proteins. Therefore, we constructed an ItfWRKY protein interaction network based on Arabidopsis homologous genes using STRING software to systematically analyse the interaction of ItfWRKY proteins (Fig. 4). Among the proteins, AtWRKY33 (ItfWRK28.1, - 28.2, - 80, - 83.1 and - 83.2), MPK4, AtWRKY29 (ItfWRKY57), ACS6, AtW RKY22 (ItfWRKY24 and ItfWRKY - 54) and PAD3 are related to the Kyoto Encyclopedia of Genes and Genomes (KEGG) signalling pathway of plant mitogen-activated protein kinases, which include cell defence response, defence response for pathogens, stress adaptation and stress tolerance. WRKY53 (ItfWRK21, - 23, - 35, - 59, - 70 and - 71) is an early factor in drought response, and it regulates stomatal movement and early events of leaf senescence by reducing $\mathrm{H}_{2} \mathrm{O}_{2}$ content and promoting starch metabolism in guard cells [46]. WRKY6 (ItfWRK3, $-11,-65,-62$, $52.1,-52.2,-52.3,-37.1$ and -37.2 ) participates in the control of aging and pathogen defence [47]. WRKY33 (Itf WRKY 28.1, $-28.2,-80,-83.1$ and -83.2 ) specifically interacts with W-box and responds to salt, cold and heat stresses [14, 48]. WRKY28 (ItfWRKY4, -12 and - 78) and AtbHLH17 confer abiotic stress resistance on A. thaliana [49]. These interacting proteins indicate that ItfWRKY proteins have similar functions to Arabidopsis proteins. The interaction network of ItfWRKYs provides new research ideas for exploring the new functions of these proteins in the future.

\section{Tissue-specific expression of ItfWRKYs in I. trifida}

To evaluate the potential functions of ItfWRKYs in plant tissue development, we studied the expression patterns of ItfWRKY genes in different tissues (flower, flower bud, root, leaf and stem). As shown in Fig. 5, the expression levels of 


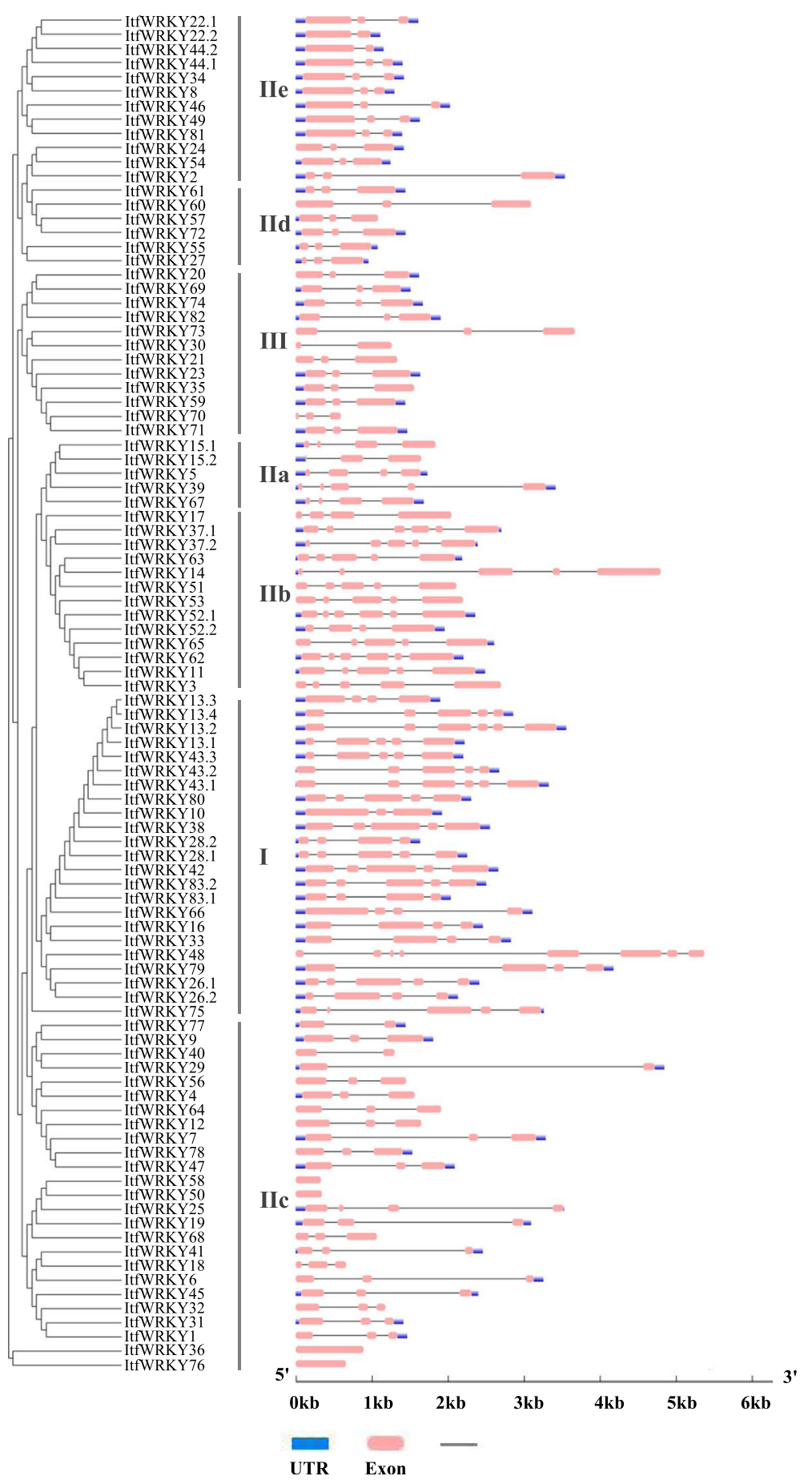

Fig. 3 Gene structure of WRKY in I. trifida. The evolutionary tree of ItfWRKY genes was constructed using MEGA7 and shown on the left. Schematic of exon/intron structure was displayed by the Gene Structure Display Server (http://gsds.cbi.pku.edu.cn/). The exons, introns and UTRs are represented by red solid boxes, black lines and blue boxes, respectively

ItfWRKYs varied among different tissues. However, some ItfWRKYs share similar expression patterns. For instance, ItfWRKY8, ItfWRKY16, ItfWRKY61, ItfWRKY66, ItfWRKY 48 and ItfWRKY79 were highly expressed in all five tissues, whereas ItfWRKY1, ItfWRKY6, ItfWRKY13.4, ItfWRKY18,
ItfWRKY30, ItfWRKY35, ItfWRKY36, ItfWRKY58, ItfWRKY 68, ItfWRKY70 and ItfWRKY73 were lowly expressed. In addition, different transcripts from the same gene were differently expressed. For example, ItfWRKY44.1 was highly expressed in flower, leaf, root and stem, whereas 


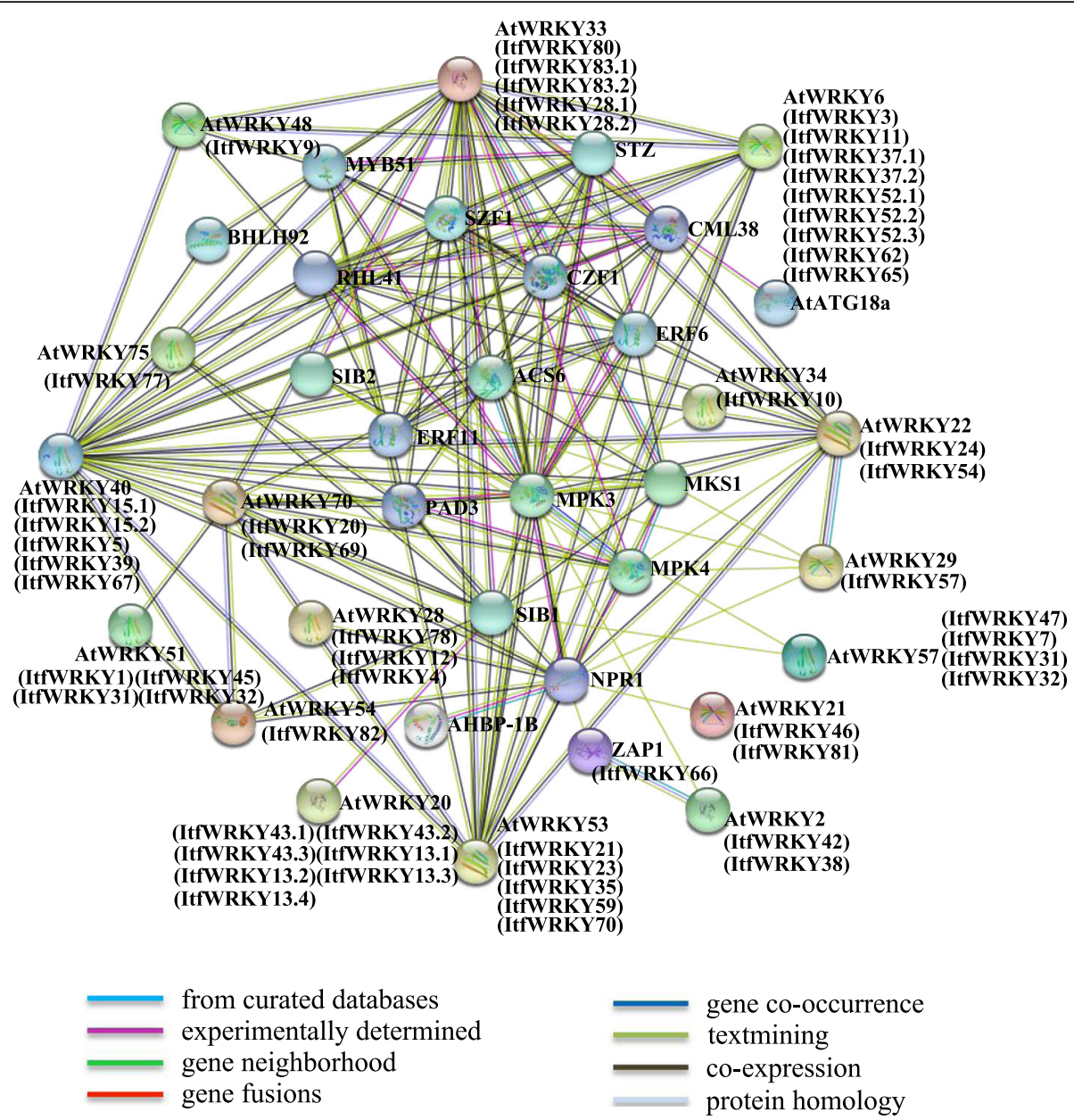

Fig. 4 Functional interaction networks of ItfWRKY proteins in I. trifida according to orthologues in A. thaliana. Network nodes represent proteins, and edges represent protein-protein associations

ItfWRKY44.2 was lowly expressed in flower, flower bud, root and stem. Most ItfWRKY genes were lowly expressed in flower and flower bud. Interestingly, ItfWRKY62, ItfWRKY67, ItfWRKY69 and ItfWRKY82 were lowly expressed in flower bud but highly expressed in flower. ItfWRKY3, ItfWRKY9, ItfWRKY12 and $I f$ fWRKY78 were expressed highly only in the roots. These results indicate ItWRKYs play diverse roles in plant tissue development. To verify the RNA-seq data of ItfWRKYs, we randomly selected 11 ItfWRKY genes and investigated their expression profiles in different tissues (flower, root, stem and leaf) by quantitative polymerase chain reaction (qPCR) (Fig. 6). The gene expression pattern from qPCR results is similar to that from transcriptome sequencing (RNA-seq) data. For instance, the expression levels of ItfWRKY8, ItfWRKY 15.1, ItfWRKY22.1, ItfWRKY48 and ItfWRKY8O were higher in the roots and stems than in the leaves and flowers (Fig. 6), suggesting that the RNA-seq data are well consistent with the qPCR results.
Stress-responsive gene expression of ItfWRKYs under different abiotic stresses

WRKYs play key roles in abiotic stress responses in plants $[50,51]$. The heat map exhibited the stress-responsive expression patterns of ItfWRKYs under salt, drought, cold and heat stresses (Fig. 7). The expression levels of 11 ItfWRKYs (ItfWRKY8, - 15.1, - 22.1, - 34, - 41, - 48, - 66, - 69, - 77, -79 and -80 ) were up-regulated under all four stress conditions, whereas those of 34 ItfWRKY genes (e.g. ItfWR $K Y 37.1,-13.1,-43.2,-50,-53,-58$ and -63.$)$ were down-regulated. The expression levels of ItfWRKY7, ItfWRKY28.1, ItfWRKY29, ItfWRKY44.1 and ItfWRKY62 increased under salt, drought and cold stresses. The expression levels of ItfWRKY11, - 45, - 52.1, - 61 and - 82 were induced under cold and heat stresses. Under heat, salt and drought stresses, the expression levels of ItfWRKY16, ItfWR KY43.1 and ItfWRKY81 increased. The expression levels of ItfWRKY $2,-4,-14,-23,-47,-59,-71,-83.1$ and 83.2 were induced by cold stress. The expression levels of ItfWRKY4, $-11,-47,-52.2$ and -71 were dramatically 


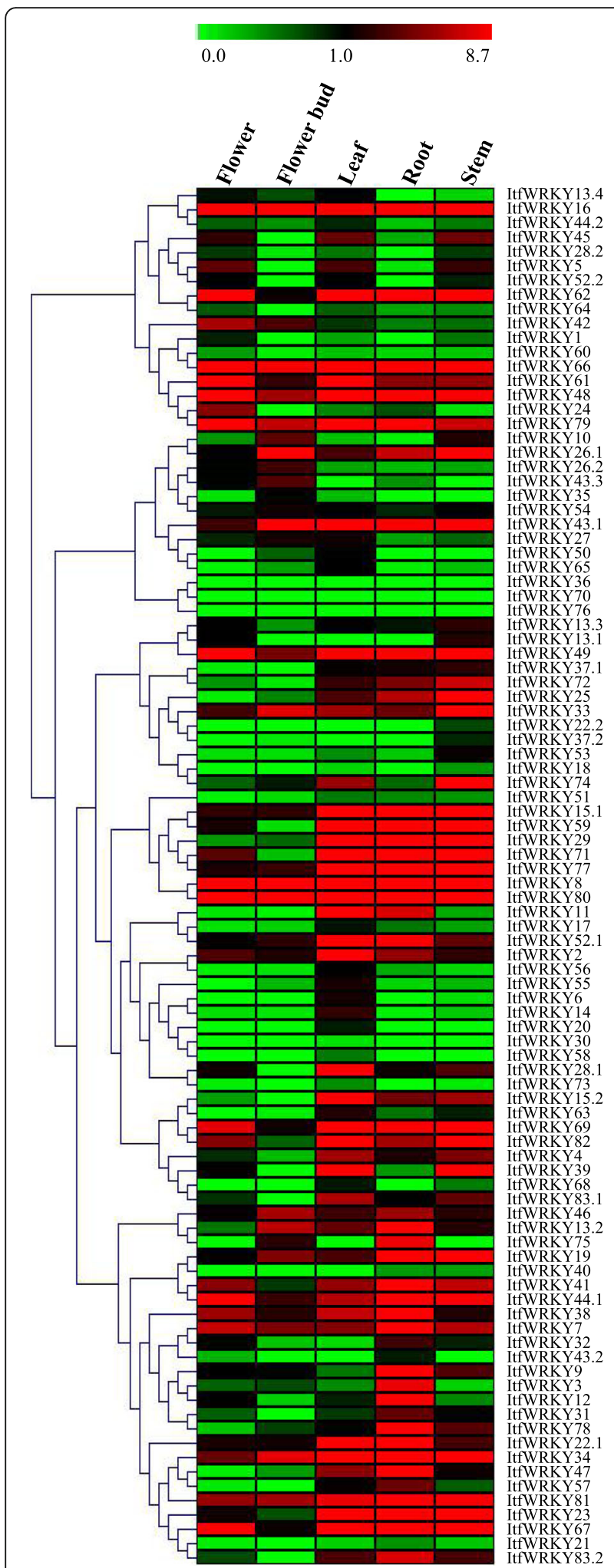

Fig. 5 Relative expression levels of ItfWRKYs across various tissues. A heat map with clustering is created based on the FPKM value of ItfWRKYs. The coloured scale varies from green to red, indicating relatively low or high expression repressed by salt; those of ItfWRKY2, -52.1 and -67 by drought; those of ItfWRKY22.2, - 28.1, - 29, - 44.1 and -62 by cold; and those of ItfWRKY13.2, $-19,-25$ and -27 by heat.

To further confirm the stress-responsive gene expression of the ItfWRKYs, we selected 11 ItfWRKY genes (ItfWRKY$8,-15.1,-22.1,-34,-41,-48,-66,-69,-77,-79$ and -80 ) and checked their expression in the roots and leaves under salt, drought, heat and cold stresses at $0,6,12$, 24 and $48 \mathrm{~h}$ time points (Fig. 8). Results showed that the ItfWRKY $Y$ genes differently responded to various stress treatments. In the roots, the expression levels of ItfWRKY22.1 and ItfWRKY34 dramatically increased at 24 and $48 \mathrm{~h}$ under salt stress, and those of ItfWRKY48 and ItfWRKY 69 were down-regulated; the expression levels of ItfWRKY22.1, $-48,-66$ and -79 were dramatically up-regulated under cold stress, and that of ItfWRKY15.1 was down-regulated; the expression of ItfWRKYY77 was dramatically up-regulated under drought stress, and those of ItfWRKY8, - 15.1 and 69 were down-regulated. The expression of most ItfWRKY genes were up-regulated by heat treatments, except ItfWRK Y15.1 and ItfWRKY77. In the leaves, ItfWRKY41 and ItfWR KY66 were highly expressed under salt stress; ItfWRKY22.1, $-34,-41,-69$ and -77 were highly expressed under drought stress; ItfWRKY66 and ItfWRKY69 were highly expressed under cold stress; and ItfWRKY8, - 22.1, - 41, 48 and -69 were highly expressed under heat stress. The up- or down-regulation of the ItfWRKYs suggests that ItfWRKYs play different roles in abiotic stress responses in I. trifida.

\section{Discussion}

Sweet potato is a staple crop with important health-care function and industrial value. Since the first WRKY protein SPF1 was isolated from sweet potato (I. batatas) [52], the WRKYs in Ipomoea species remain largely unknown. Considering the complicated genetic background of sweet potato due to its hexaploid character, we carried out the genome-wild identification and characterisation of WRKYs in I. trifida, the wild ancestor of sweet potato. WRKY as an important TF can rapidly increase its family members to establish a signal transduction network in adversity, which may optimise plant adaptability [53]. Several WRKY genes have been identified in plants, such as 72 WRKY genes in A. thaliana [54], 109 in Oryza sativa [20], 197 in Glycine max [4], 80 in Pinus monticola [55], 35 in Selaginella involucrate [56], 45 in Hordeum vulgare [41], 38 in Physcomitrella patens, 104 in Populus spp., 68 in Sorghum bicolor and 66 in Carica papaya [6]. In the present study, 83 ItfWRKY genes encoding 96 transcripts were identified in I. trifida.

Segmental duplication of chromosome regions results in a scattered pattern of occurrence, and tandem duplication results in a clustered pattern [57]. Both gene duplication 

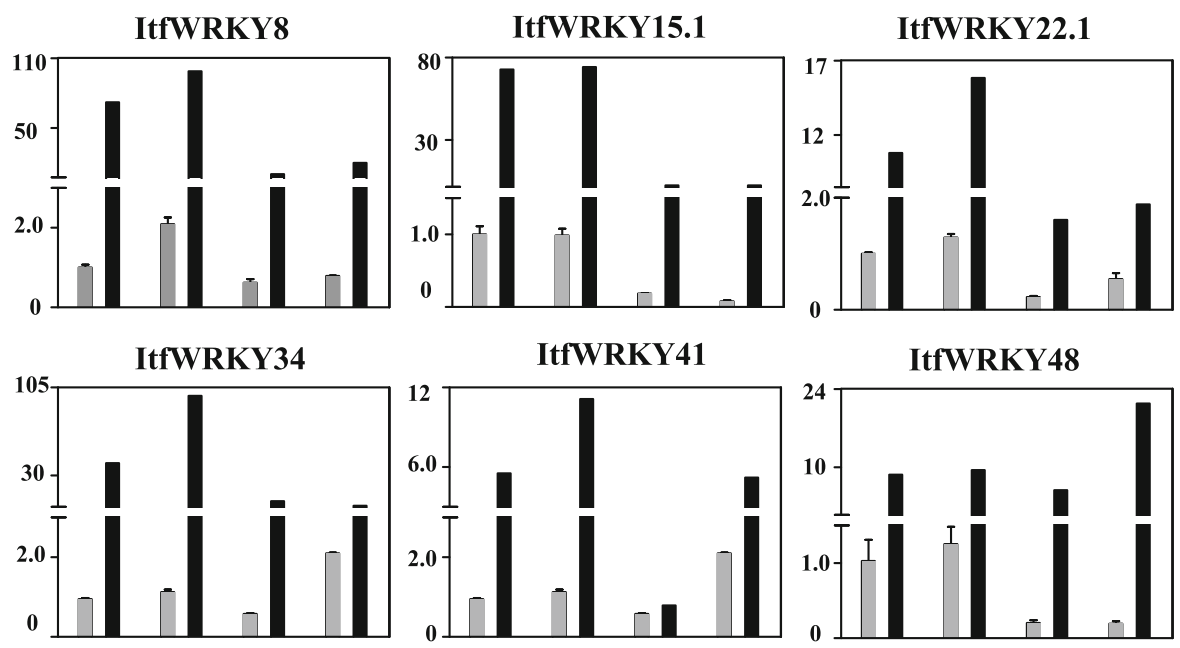

ItfWRKY41

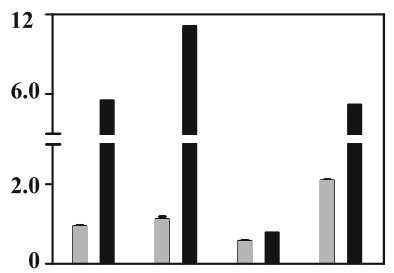

ItfWRKY48

ItfWRKY66

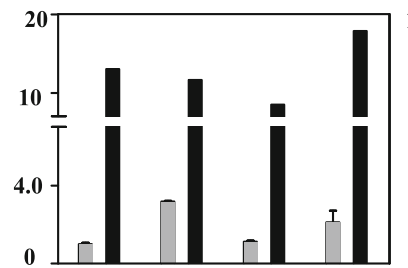

ItfWRKY79

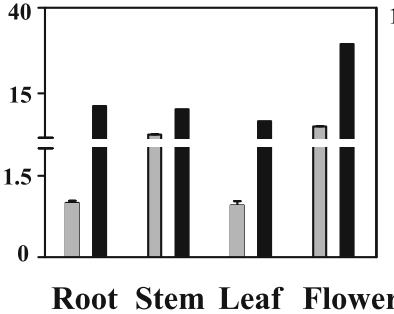

ItfWRKY69

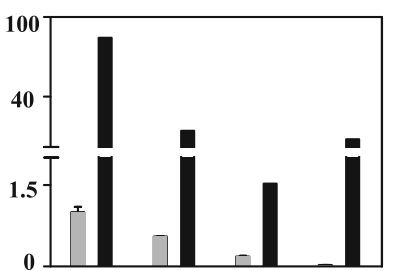

ItfWRKY80

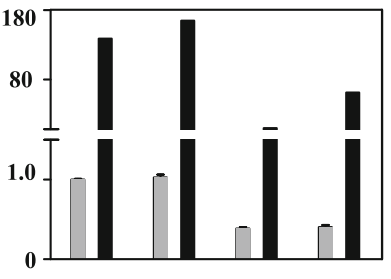

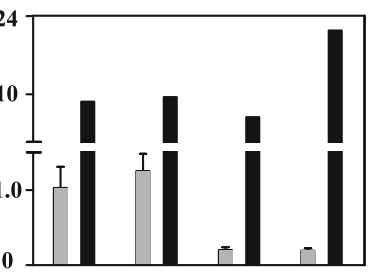

ItfWRKY77

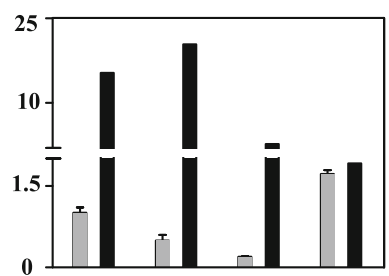

Root Stem Leaf Flower

\section{qRT-PCR}

RNA-seq

Fig. 6 Comparison between quantitative RT-PCR data and RNA-seq data. Relative expression of the 11 selected ItfWRKYs was analysed by qRTPCR. The GAPDH transcript levels were used for normalisation. The $y$-axis represents the relative expression of the fold. Error bars indicate standard deviation. RNA-seq and GRT-PCR data are represented by black and grey bars, respectively

has been observed in the WRKY TF family in A. thaliana $[58,59]$, rice $[56,60,61]$, tomato [62] and cucumber [63, 64]. However, tandem gene duplication was not observed in I. trifida in our study (Fig. 1), which is similar to the previous report in sesame (Sesamum indicu) $[65,66]$. A total of 50 segmental duplications was identified in the WRKY gene family in I. trifida. In addition, 4 genes (ItfWRKY35, ItfWRKY69, ItfWRKY70 and ItfWRKY71) in Group III and 6 genes (ItfWRKY10, ItfWRKY26, ItfWRK Y48, ItfWRKY66, ItfWRKY75 and ItfWRKY79) in Group I do not undergo segmental duplication events. At the same time, ItfWRKYs in Group IIa had no segmental duplication, suggesting that gene duplication did not occur in Group IIa. The above results indicated that segmental duplication is very important for WRKY gene family expansion and evolution in I. trifida.
In general, intron distributions can be used to study gene evolution, and genes containing many introns are usually considered conservative. The number of ItfWRKY introns ranges from 0 to 7. Among them, ItfWRKY13.2, - 37.1, 43.1, -52.1 and -62 have 5 introns, and ItfWRKY48 has 7 introns (Fig. 3), indicating that these ItfWRKYs are highly conserved during evolution. Most ItfWRKYs have a highly conserved WRKY (WRKYGQK) domain, whereas individual ItfWRKYs show some substitutions, such as glutamine being replaced by lysine residues (ItfWRKY1, - 6, - 18, $31,-32,-45$ and 76 ), glutamine being replaced by threonine residues and lysine being replaced by arginine residues (ItfWRKY36) (Additional file 2: Fig. S1), which is consistent with previous findings [3, 40-42].

To understand the potential function of ItfWRKYs, we analysed the tissue-specific expression pattern in the 


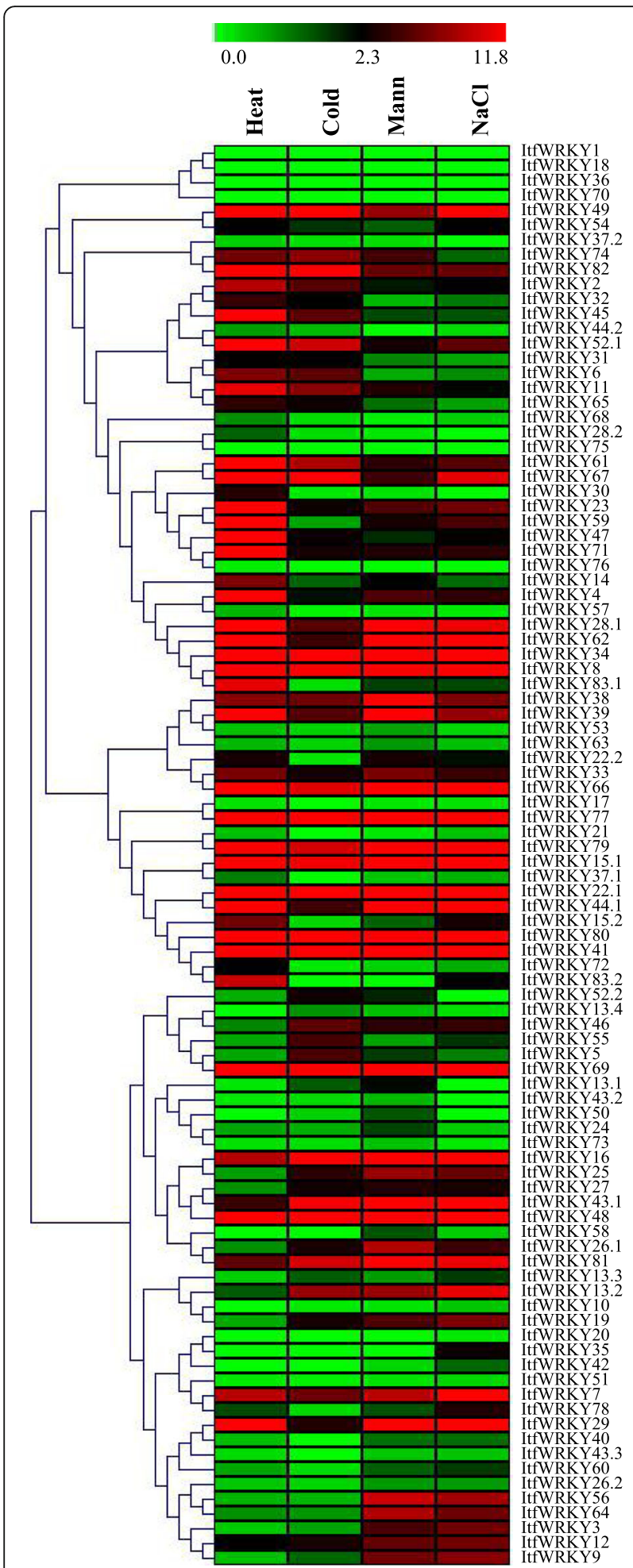

Fig. 7 Responses of ItfWRKYs to adverse environmental stresses. Expression profiles under four abiotic stresses (cold, heat, drought and salt) were analysed. The coloured scale varies from green to red, which indicates the low or high expression of each gene leaves, roots, stems, flowers and flower buds and the stress-responsive expression pattern under four abiotic stresses in I. trifida. Most ItfWRKY genes are highly expressed in the roots and leaves, whereas a few are expressed in flowers, which is similar to the findings of ZmWRKY, AtWRKY, OsWRKY and VvWRKY family members [67]. Thirty-four ItfWRKYs were highly expressed in at least three tissues in I. trifida. Fifteen of these genes (e.g. ItfWRKY8, ItfWRKY16, ItfWRKY48, Itf WRKY49, ItfWRKY66 and ItfWRKY80) are highly expressed in all tissues, suggesting that these highly expressed WRKYs are important regulatory factors for tissue development in I. trifida. Most of these highly expressed ItfWRKYS are in the Group I and II subfamilies. A previous study has shown that Group I WRKYS, as the ancestors of other WRKYs, are expressed constitutively in different tissues [68]. For example, the Group I ItfWRKYs (ItfWRKY66, - 69 and - 80) are expressed in most I. trifida tissues and highly expressed under cold, heat and drought stress conditions. By contrast, the expression levels of 12 ItfWRKYs (ItfWRKY18, - 21, - 30, $-36,-40,-51,-58,-60,-64,-70,-73$ and -76 ) were low in all $I$. trifida tissues, and two ItfWRKYs (ItfWRKY30 and ItfWRKY58) were only expressed in one tissue. These lowly expressed ItfWRKYs are distributed in all WRKY subgroups, and most of them are in Groups IIc and IIe. The IIc WRKYs in A. thaliana (such as AtWRKY8, -48, - 50 and -57) are involved in pathogen responses and jasmonic acid (JA)- and salicylic acid (SA)-mediated signalling pathways [48]. Thus, we implied that IIc ItfWRKYS with low expression levels in most tissues may function in pathogen responses rather than tissue development.

To evaluate the potential functions of ItfWRKYs, we summarised the known functions of WRKY under abiotic stress and compared the gene expression between functional known WRKYs and their I. trifida homologs (Table 1). As shown in Table 1, the functions of some ItfWRKYs homologous genes have been characterised in $A$. thaliana, T. aestivum, O. sativa, G. max, G. hirsutum and other species under various stresses, including cold, salt, heat and drought stresses [14-21, 23-28, 69-75]. For example, overexpression of AtWRKY25 enhances heat and salt tolerance in Arabidopsis [14], and its homologous ItfWRKY28.1 is induced by heat and salt stresses, indicating that ItfWRKY28.1 plays the same role under heat and salt stresses. Moreover, similar patterns were investigated for most reported Arabidopsis WRKYS and their homologous ItfWRKYs, such as AtWRKY26 and ItfWRKY43.1, AtWRK Y33 and ItfWRKY13.3, AtWRKY39 and ItfWRKY44.2, and AtWRKY57 and ItfWRKY4. However, AtWRKY34 is a negative regulator in pollen specific cold response, but the expression of its homologous ItfWRKY38 is up-regulated under cold stress, suggesting that ItfWRKY38 plays a 

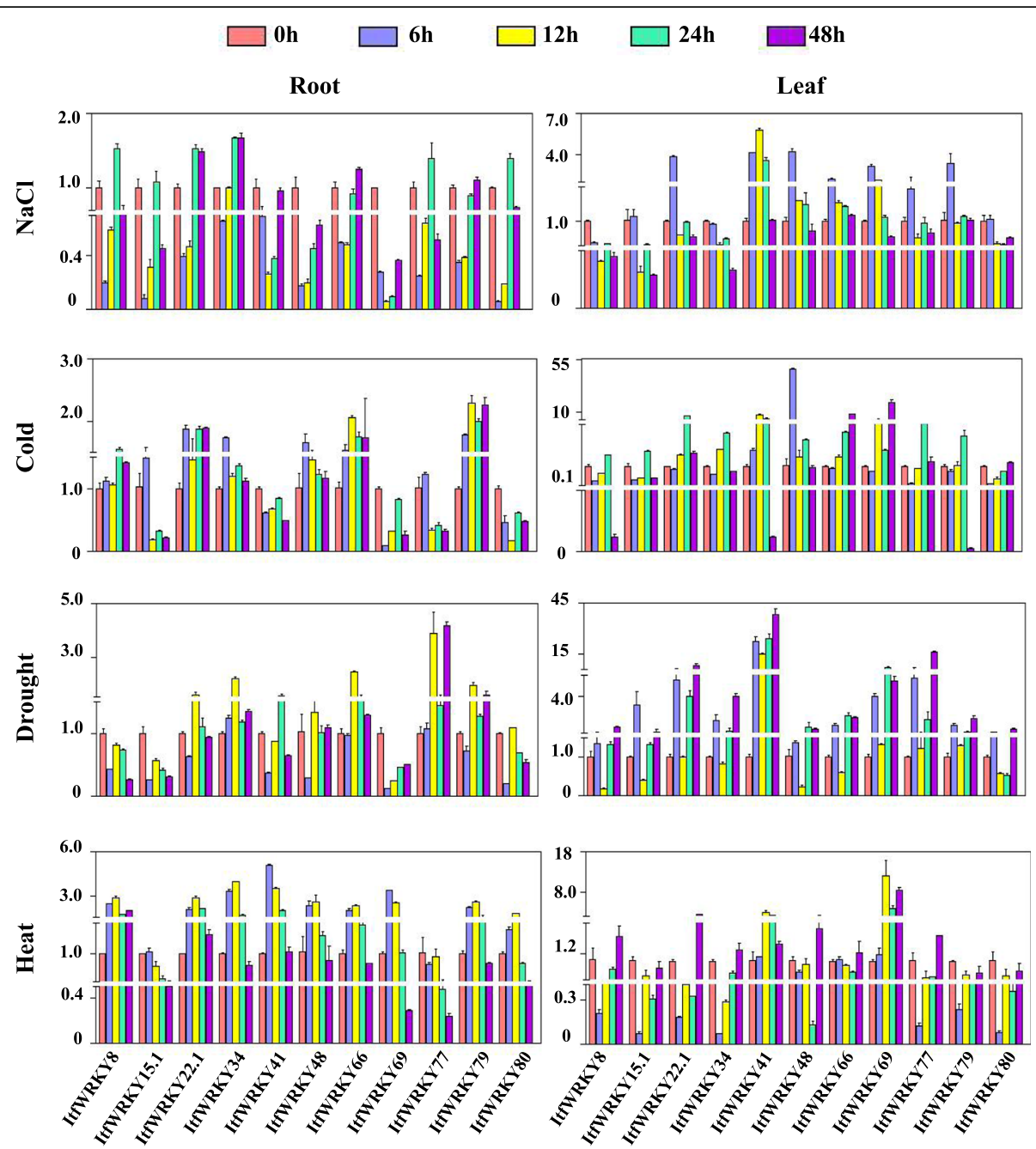

Fig. 8 Gene expression confirmed by qRT-PCR under abiotic stresses. The expression at $0 \mathrm{~h}$ was set up as 1 fold. The $y$-axis indicates the fold changes of relative gene expression compared with the expression at $0 \mathrm{~h}$. Error bars indicate standard deviation. The expression levels at 0, 6, 12, 24 and $48 \mathrm{~h}$ are indicated by red, blue, yellow, green and purple bars, respectively

negative role specific in pollen and a positive role in the other tissues under cold stress. For the other homologous ItfWRKYs, most of their gene expression patterns were consistent with those of the functional known WRKYs (Table 1). Taken together, the regulation of WRKYs contributes to its crucial roles in plant abiotic stress responses, which may further establish the complex signalling networks for stress tolerance and adaptation in I. trifida.

\section{Conclusion}

In this study, we identified 83 ItfWRKY genes encoding 96 WRKY TFs and investigated their gene distribution, structure and evolutionary characteristics. The tissue-specific and stress-responsive expression patterns of ItfWRKYs showed that these genes play important roles in plant development, abiotic stress response and adaptation. Our study has established the functional framework of
ItfWRKYs, which can facilitate the further functional studies of WRKYs and the molecular breeding of sweet potato.

\section{Methods}

\section{Data collection and ItfWRKY identification}

The sequences of ItfWRKYs were obtained from the Sweet potato Genomics Resource (http://sweetpotato. plantbiology.msu.edu/), and those of A. thaliana WRKY were downloaded from TAIR (https://www.arabidopsis. org/.jsp). The data are shown in Additional file 1: Tables S1. In this study, 114 putative WRKY TFs were retrieved from the sweet potato genome database. The PFAM (http://pfam.xfam.org/) and CDD (http://www.ncbi.nlm. nih.gov/cdd/) databases were used to further confirm whether or not these sequences contain the WRKY domain. Finally, 96 WRKY TFs encoded by 83 WRKY genes were identified for this study. 


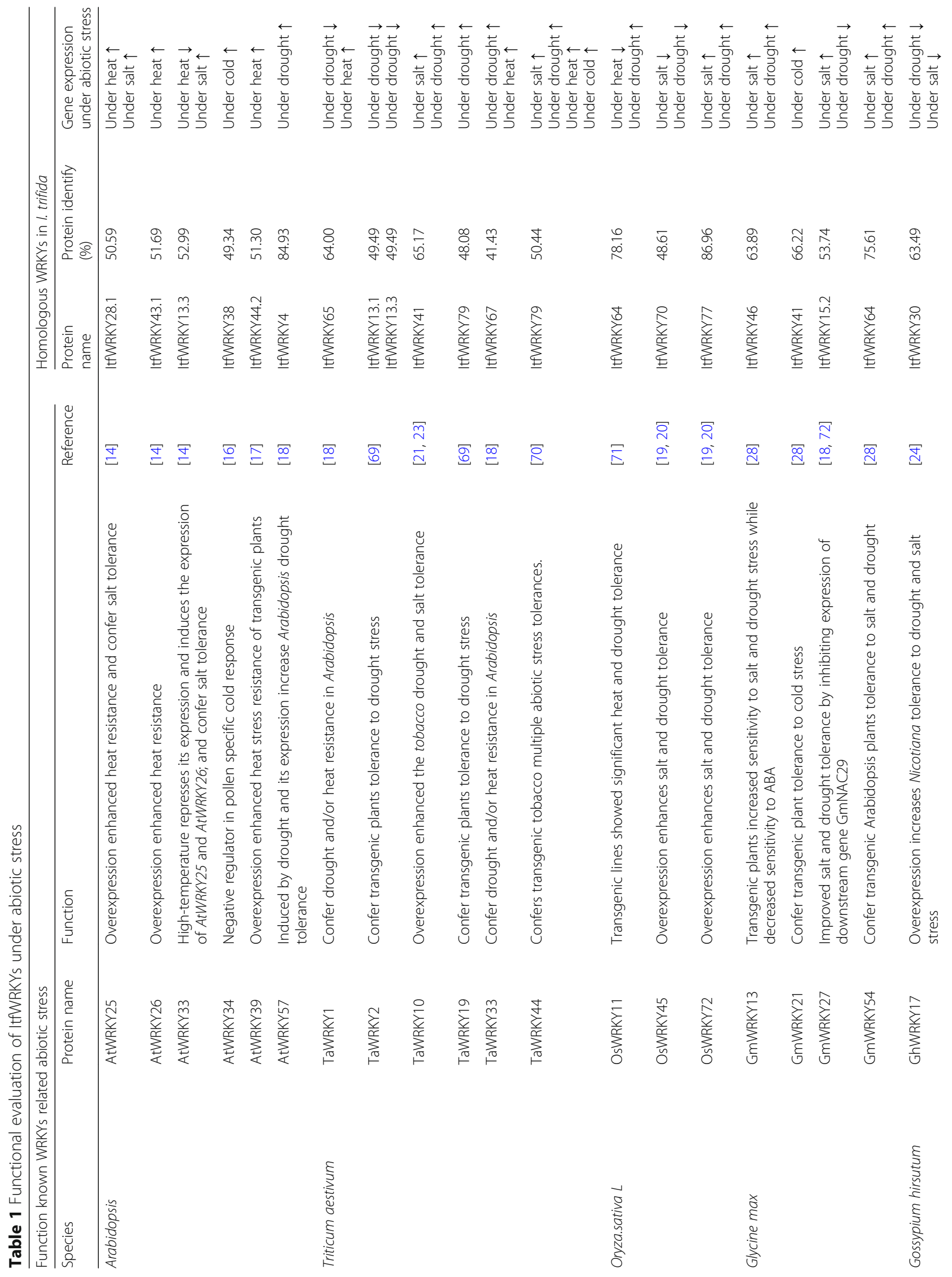




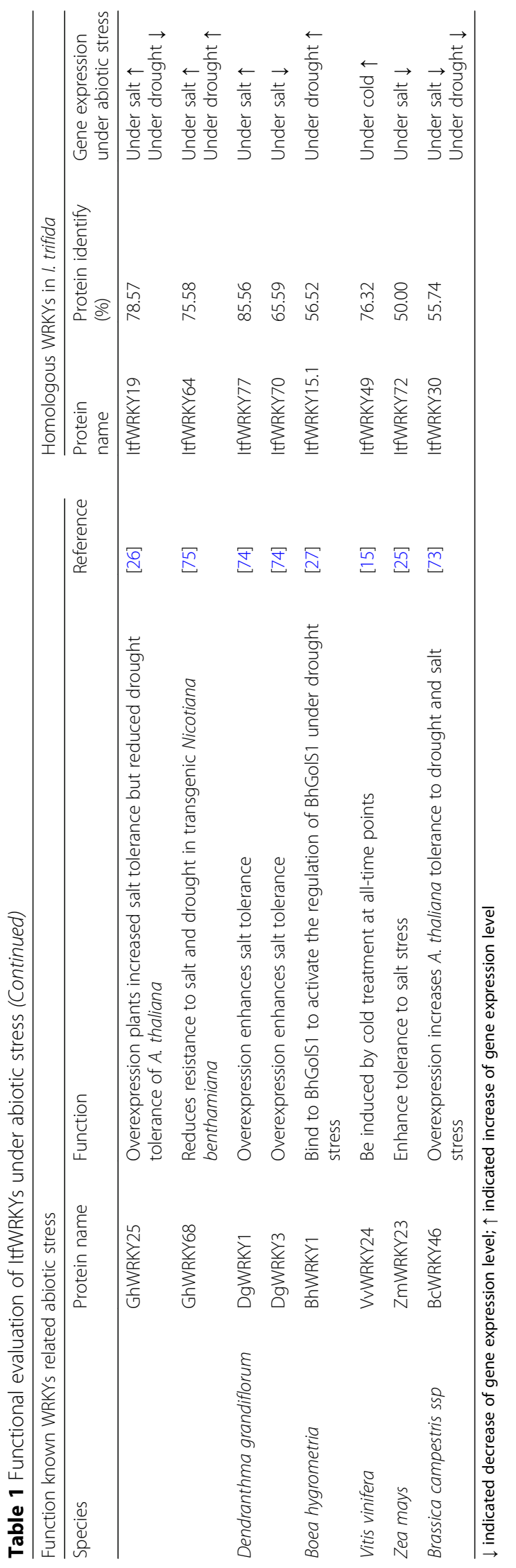




\section{Chromosomal distribution and gene duplication analysis of ItfWRKYs}

On the basis of the chromosomal location data provided by the database (http://sweetpotato.Plantbiology.msu. $\mathrm{Edu} /$ ), the ItfWRKYs were mapped on the chromosome of I. trifida. Gene duplication were analysed by the Multiple Collinearity Scan toolkit and visualised by Circos (http://circos.ca/) [22, 76].

\section{Gene structure analysis and motif composition of ItfWRKYs}

The gene structure of ItfWRKY was determined by comparing their genomic sequences with predicted coding sequences using the Gene Structure Display Server (http:// gsds.cbi.pku.edu.cn/). The conserved motifs in the ItfWRKY proteins were analysed by the online programmer MEME (http://meme-suite.org/tools/meme).

\section{Protein properties and phylogenetic tree construction}

The molecular weight (MW) and isoelectric points (pIs) of the ItfWRKY proteins were determined using the ProtParam program (ExPASy tools) (http://expasy.org/ tools/). Meanwhile, phosphorylation analysis and subcellular localisation prediction were carried out using the P3DB online tool (http://www.p3db.org/) and the software WoLFPSORT (https://wolfpsort.hgc.jp/) forecast, respectively. The neighbour-joining (NJ) phylogenetic trees of I. trifida and A. thaliana WRKY proteins were generated using MEGA7 [77]. In addition, a protein network of functional interactions between $I$. trifida and $A$. thaliana was constructed by STRING software (https:// string-db.org/cgi/network.pl).

\section{Transcriptome and quantitative real-time polymerase chain reaction (PCR) analysis}

The ItfWRKY RNA-seq data (in Additional file 5: Table S4 and Additional file 6: Table S5) were downloaded from the sweet potato database (http://sweetpotato. plantbiology.msu.edu/). The gene expression levels of ItfWRKYs were calculated as fragments per kilobase of exon per million fragments mapped (FPKM). The heat maps of ItfWRKY expression profiles were generated using Mev.4.9.0. Total RNA was isolated by using the RNAprep Pure Kit (Tiangen Biotechnology, Beijing, China). The first chain of DNA was synthesised by PrimeScript $^{\mathrm{TM}}$ RT Reagent Kit (Tsingke, Nanjing, China) and used as the template for quantitative PCR. The primers used for PCR are listed in Additional file 7: Table S6, and qPCR was carried out on ABI Step One Plus instrument (Biosoar, Nanjing, China).

\section{Plant growth and stress treatments}

I. trifida plants were collected from Xuzhou Academy of Agricultural Science. The plants were grown as previously described [78]. In brief, vermiculite, perlite and soil were mixed at a ratio of 1:1:4 and placed under $16 \mathrm{~h}$ light $/ 8 \mathrm{~h}$ darkness and $26^{\circ} \mathrm{C}$ growth temperature. To study the tissue-specific patterns of ItfWRKYs, we collected the roots, leaves and stems of four-week-old I. trifida seedlings and flowers of two-month-old plants [39]. For the analysis of stress-responsive expression patterns, fourweek-old plants were divided into five treatment groups: control group, salinity treatment group (200 mM NaCl solution), cold treatment group $\left(12{ }^{\circ} \mathrm{C}\right)$, drought treatment group (300 $\mathrm{mM}$ mannitol solution) and heat treatment group $\left(40^{\circ} \mathrm{C}\right)$. Leaves and roots were sampled at $0,6,12$, 24 and $48 \mathrm{~h}$.

\section{Supplementary information}

Supplementary information accompanies this paper at https://doi.org/10. 1186/s12863-019-0789-x.

Additional file 1: Table S1. Accession numbers of WRKY genes in Ipomoea trifida and Arabidopsis thaliana.

Additional file 2: Fig S1. Alignment of ItfWRKY domain sequences. The alignment was performed by the Multiple interface page (http://multalin. toulouse.inra.fr/multalin/). The conserved WRKY aa and zinc-finger motifs are highlighted in red. Gaps are indicated by dashes.

Additional file 3: Table S2. Informations of ItfWRKYs.

Additional file 4: Table S3. Chromosomal locations and segmental duplication of ItfWRKY genes.

Additional file 5: Table S4. Relative expression levels of ItfWRKYs in various tissues.

Additional file 6: Table S5. Expression pattern of ItfWRKYs under abiotic stresses.

Additional file 7: Table S6. Primers of the ItfWRKY genes and housekeeping gene for QRT-PCR.

\section{Abbreviations}

BLASTP: Protein-protein BLAST; Chr: Chromosome; JA: Jasmonic acid; kDa: Kilodalton; KEGG: Kyoto Encyclopedia of Genes and Genomes; MCSCANX: Multiple Collinearity Scan tool kit X version; NJ: Neighbourjoining; pl: Isoelectric point; Protein MW: Protein molecular weigh; qPCR: quantitative polymerase chain reaction; RNA-seq: Transcriptome sequencing; SA: Salicylic acid; TF: Transcription factor; WRKY: WRKY DNAbinding protein

\section{Acknowledgments}

We thank the GT4SP project team for sharing the Ipomoea trifida genome annotation data (http://sweetpotato.plantbiology.msu.edu/).

\section{Author's contributions}

TX conceived and designed this experiment. YL, LZ and PZ carried out the experiments and analyzed the data. QC offered the plant material. JS helped to analyze the data. TX and YL wrote the manuscript. QC and ZY helped to revise the manuscript. All authors read and approved the manuscript.

\section{Funding}

This work was supported jointly by the projects of the National Natural Science Foundation of China (Grant No. 31701481), the Natural Science Foundation of Jiangsu Higher Education Institutions of China (19KJA510010), the Priority Academic Program Development of Jiangsu Higher Education Institutions (No. PAPD), the China Agriculture Research System (CARS-10B03), Jiangsu Overseas Visiting Scholar Program for University Prominent Young \& Middle-aged Teachers and Presidents, and National Key R\&D Program of China (2018YFD1000705, 2018YFD1000700). These funding bodies supported this research from inception to completion, including the 
experiment design, data collection, analysis, interpretation, and the manuscript writing.

\section{Availability of data and materials}

All data generated or analysed during this study are included in this published article and its additional files.

\section{Ethics approval and consent to participate}

Not applicable

\section{Consent for publication}

Not applicable

\section{Competing interests}

The authors declare they have no competing interests.

\begin{abstract}
Author details
${ }^{1}$ Key lab of phylogeny and comparative genomics of the Jiangsu province, Institute of Integrative Plant Biology, School of Life Sciences, Jiangsu Normal University, Xuzhou 221116, Jiangsu Province, China. ${ }^{2}$ Department of Plant Biotechnology, College of Agriculture and Life Sciences, Chonnam National University, Gwangju 500-757, South Korea. ${ }^{3}$ Xuzhou Academy of Agricultural Sciences/Sweet Potato Research Institute, CAAS, Xuzhou 221121, Jiangsu, China.
\end{abstract}

Received: 12 August 2019 Accepted: 14 November 2019 Published online: 03 December 2019

\section{References}

1. Chen L, Song Y, Li SJ, Zhang LP, Zou CS, Yu DQ. The role of WRKY transcription factors in plant abiotic stresses. Biochim Biophys Acta. 2012; 1819(2):0-128

2. Chinnusamy V, Schumaker K, Zhu JK. Molecular genetics perspectives on cross-talk and specificity in abiotic stress signalling in plants. J Exp Bot. 2004; 55:225-36.

3. Eulgem T, Rushton PJ, Robatzek S, Somssich IE. The WRKY superfamily of plant transcription factors. Trends Plant Sci. 2000;5:199-206.

4. Schmutz J, Cannon SB, Schlueter J, Ma J, Mitros T, Nelson W, Hyten DL, Song Q, Thelen JJ, Cheng J, Xu D, Hellsten U, May GD, Yu Y, Sakurai T, Umezawa T, Bhattacharyya MK, Sandhu D, Valliyodan B, Lindquist E, Peto M, Grant D, Shu S, Goodstein D, Barry K, Futrell-Griggs M, Abernathy B, Du J, Tian Z, Zhu L, Gill N, Joshi T, Libault M, Sethuraman A, Zhang XC, Shinozaki K, Nguyen HT, Wing RA, Cregan P, Specht J, Grimwood J, Rokhsar D, Stacey G, Shoemaker RC, Jackson SA. Genome sequence of the palaeopolyploid soybean. Nature. 2010;463:178-83.

5. Bakshi M, Oelmüller R. WRKY transcription factors. Plant Signal Behav. 2014 9:e27700.

6. Pandey SP, Somssich IE. The role of WRKY transcription factors in plant immunity. Plant Physiol. 2009;150:1648-55

7. Xie Z, Zhang ZL, Zou X, Huang J, Ruas P, Thompson D, Shen QJ. Annotations and functional analyses of the rice WRKY gene superfamily reveal positive and negative regulators of abscisic acid signaling in aleurone cells. Plant Physiol. 2005:137:176-89.

8. Johnson CS, Kolevski B, Smyth DR. TRANSPARENT TESTA GLABRA2, a trichome and seed coat development gene of Arabidopsis, encodes a WRKY transcription factor. Plant Cell. 2002;14:1359-75.

9. Song $Y, A i C R$, Jing SJ, Yu DQ. Research progress on function analysis of rice WRKY gene. Rice Sci. 2010;17:60-72.

10. Xu YH, Wang JW, Wang S, Wang JY, Chen XY. Characterization of GaWRKY1, a cotton transcription factor that regulates the sesquiterpene synthase gene (+)-delta-cadinene synthase-a. Plant Physiol. 2004;135:507-15.

11. Lagace M, Matton DP. Characterization of a WRKY transcription factor expressed in late torpedo-stage embryos of Solanum chacoense. Planta. 2004:219:185-9.

12. Miao Y, Zentgraf $U$. The antagonist function of Arabidopsis WRKY55 and ESR/ESP in leaf senescence is modulated by the jasmonic and salicylic acid equilibrium. Plant Cell. 2007;19:819-30.

13. Zhou X, Jiang YJ, Yu DQ. WRKY22 transcription factor mediates darkinduced leaf senescence in Arabidopsis. Mol Cells. 2011;31:303-13.
14. Li SJ, Fu QT, Chen LG, Huang WD, Yu DQ. Arabidopsis thaliana WRKY25, WRKY26, and WRKY33 coordinate induction of plant thermotolerance. Planta. 2011;233:1237-52.

15. Wang M, Vannozzi A, Wang G, Liang $Y H$, Tornielli GB, Zenoni S, Cavallini $E_{\text {, }}$ Pezzotti M, Cheng ZM. Genome and transcriptome analysis of the grapevine (Vitis vinifera L.) WRKY gene family. Horti Res. 2014b;1:14016.

16. Zou C, Jiang W, Yu D. Male gametophyte-specific WRKY34 transcription factor mediates cold sensitivity of mature pollen in Arabidopsis. J Exp Bot. 2010;61:3901-14.

17. Li S, Zhou X, Chen L, Huang W, Yu D. Functional characterization of Arabidopsis thaliana WRKY39 in heat stress. Mol Cells. 2010b;29:475-83.

18. He GH, Xu JY, Wang YX, Liu JM, Li PS, Chen M, Ma YZ, Xu ZS. Drought-responsive WRKY transcription factor genes TaWRKY1 and TaWRKY33 from wheat confer drought and/orheat resistance in Arabidopsis. BMC Plant Bio. 2016;116:116.

19. Oiu YP, Yu DO. Over-expression of the stress-induced OsWRKY45 enhances disease resistance and drought tolerance in Arabidopsis. Environ Exp Bot. 2009;65:35-47.

20. Song Y, Chen LG, Zhang LP, Yu DQ. Overexpression of OsWRKY72 gene interferes in the abscisic acid signal and auxin transport pathway of Arabidopsis. J Biol Sci. 2010b;35:459-71.

21. Jiang JJ, Ma SH, Ye NH, Jiang M, Cao JS, Zhang JH. WRKY transcription factors in plant responses to stresses. J Integr Plant Biol. 2016;59(2):86.

22. Wang Y, Tang H, DeBarry JD, Tan X, Li J, Wang X, Lee TH, Jin H, Marler B, Guo $\mathrm{H}$, Kissinger JC, Paterson AH. MCScanX: a toolkit for detection and evolutionary analysis of gene synteny and collinearity. Nucleic Acids Res. 2012;40(7):e49.

23. Wang C, Deng P, Chen L, Wang X, Ma H, Hu W, Yao N, Feng Y, Chai R, Yang G, He G. A wheat WRKY transcription factor TaWRKY10 confers tolerance to multiple abiotic stresses in transgenic tobacco. PLoS ONE. 2013;8:e65120.

24. Yan $\mathrm{H}$, Jia H, Chen $X$, Hao L, An H, Guo X. The cotton WRKY transcription factor GhWRKY17 functions in drought and salt stress in transgenic Nicotiana benthamiana through ABA signaling and the modulation of reactive oxygen species production. Plant Cell Physiol. 2014;55:2060-76.

25. Jiang WB, Yu DQ. Arabidopsis WRKY2 transcription factor may be involved in osmotic stress response. Acta Bot Yunnan. 2009:31:427-32.

26. Liu XF, Song YZ, Xing FY, Wang N, Wen FJ, Zhu CX. GhWRKY25, a group I WRKY gene from cotton, confers differential tolerance to abiotic and biotic stresses in transgenic Nicotiana benthamiana. Protoplasma. 2016;253:1265-81.

27. Wang Z, Zhu Y, Wang LL, Liu X, Liu YG, Phillips J, Deng X. A WRKY transcription factor participates in dehydration tolerance in Boea hygrometrica by binding to the $\mathrm{W}$-box elements of the galactinol synthase (BhGolS1) promoter. Planta. 2009;230:1155-66.

28. Zhou QY, Tian AG, Zou HF, Xie ZM, Lei G, Huang J, Chen SY. Soybean WRKY-type transcription factor genes, GmWRKY13, GmWRKY21, and GmWRKY54, confer differential tolerance to abiotic stresses in transgenic Arabidopsis plants. Plant Biotechnol J. 2009:6:486-503.

29. Luo X, Bai X, Sun XL, Zhu D, Liu BH, Ji W, Hu MR, Liu X, Tang LL, Zhu YM. Expression of wild soybean WRKY20 in Arabidopsis enhances drought tolerance and regulates ABA signalling. J Exp Bot. 2013;64:2155-69.

30. Zang N, Zhai H, Gao S, Chen W, He SZ, Liu QC. Efficient production of transgenic plants using the bar gene for herbicide resistance in sweet potato. Sci Hortic. 2009:122(4):649-53.

31. Xiao BZ, Chen X, Xiang CB, Tang N, Zhang QF, Xiong LZ. Evaluation of seven function-known candidate genes for their effects on improving drought resistance of transgenic rice under field conditions. Mol Plant. 2009;2(1):73-83.

32. Liu DG, He SZ, Song XJ, Zhai H, Liu N, Zhang DD, Ren ZT, Liu QC. IbSIMT1, a novel salt-induced methyltransferase gene from Ipomoea batatas, is involved in salt tolerance. Plant Cell Tissue Organ Cult. 2015;120(2):701-15.

33. Fan WJ, Deng GF, Wang HX, Zhang HX, Zhang P. Elevated compartmentalization of $\mathrm{Na}+$ into vacuoles improves salt and cold stress tolerance in sweet potato (Ipomoea batatas). Physiol Plant. 2015;154(4):560-71.

34. Zhai H, Wang FB, Si ZZ, Huo JX, Xing L, An YY, He SZ, Liu QC. A myoinositol-1-phosphate synthase gene, IbMIPS1, enhances salt and drought tolerance and stem nematode resistance in transgenic sweet potato. Plant Biotechnol. 2015:36:830-41.

35. Kang L, Kim HS, Kwon YS, Ke Q, Ji CY, Park SC, Lee HS, Deng X, Kwak SS $\mathrm{IbOr}$ regulates photosynthesis under heat stress by stabilizing IbPsbP in sweet potato. Front Plant Sci. 2017:8:989.

36. Rajapakse S, Nilmalgoda SD, Molnar M, Ballard RE, Austin DF, Bohac JR Phylogenetic relationships of the sweet potato in Ipomoea series Batatas (Convolvulaceae) based on nuclear beta-amylase gene sequences. Mol Phylogenet Evol. 2004;30(3):623-32. 
37. Huang JC, Sun M. Genetic diversity and relationships of sweet potato and its wild relatives in Ipomoea series Batatas (Convolvulaceae) as revealed by inter-simple sequence repeat (ISSR) and restriction analysis of chloroplast DNA. Theor Appl Genet. 2000;100(7):1050-60.

38. Zhang A, Dai XB, Zhou ZL, Zhao DL, Tang J, Cao QH. Research advance in self-incompatibility of Ipomoea trifida, an ancestor of sweet potato. Acta Agriculturae Jiangxi. 2017;29(5):17-21.

39. Yang ZM, Sun J, Chen Y, Zhu PP, Zhang L, Wu SY, Ma DF, Cao QH, Li ZY, Xu T. Genome-wide identification, structural and gene expression analysis of the bZIP transcription factor family in sweet potato wild relative Ipomoea trifida. BMC Genet. 2019;20:41.

40. Meng D, Li YY, Bai Y, Li MJ, Cheng LL. Genome-wide identification and characterization of WRKY transcriptional factor family in apple and analysis of their responses to waterlogging and drought stress. Plant Physiol Biochem. 2016;103:71-83.

41. Mangelsen E, Kilian J, Berendzen KW, Kolukisaoglu ÜH, Harter K, Jansson C, Wanke D. Phylogenetic and comparative gene expression analysis of barley (Hordeum vulgare) WRKY transcription factor family reveals putatively retained functions between monocots and dicots. BMC Genomics. 2008;9(1): 194.

42. Huang SX, Gao YF, Liu JK, Peng XL, Niu XL, Fei ZJ, Cao SQ, Liu YS. Genomewide analysis of WRKY transcription factors in Solanum lycopersicum. Mol Gen Genomics. 2012;287(6):495-513.

43. Zhu Y, Wu N, Song W, Yin G, Qin Y, Yan Y, Hu Y. Soybean (Glycine max) expansin gene superfamily origins: segmental and tandem duplication events followed by divergent selection among subfamilies. BMC Plant Biol. 2014;14(1):93.

44. Yu J, Wang J, Lin W, Li SZ, Li H, Zhou J, Ni PX, Dong W, Hu SN, Zeng CQ, Zhang JG, Zhang Y, Li RQ, Xu ZY, Li ST, Li XR, Zheng HK, Cong LJ, Lin LY, Yin JN, Geng JN, Li GY, Shi JP, Liu J, Lv H, Li J, Wang J, Deng YJ, Ran LH, Shi $X \mathrm{~L}$. The genomes of Oryza sativa: a history of duplications. PLoS Biol. 2005; 3(2):e38.

45. Ramamoorthy R, Jiang SY, Kumar N, Venkatesh PN, Ramachandran S. A comprehensive transcriptional profiling of the WRKY gene family in rice under various abiotic and phytohormone treatments. Plant Cell Physiol. 2008:49(6):865-79.

46. Sun YD, Yu DQ. Activated expression of AtWRKY53 negatively regulates drought tolerance by mediating stomatal movement. Plant Cell Rep. 2015; 34(8):1295-306.

47. Huang YY, Zhang LL, Ma XF, Zhao ZX, Zhao JH, Zhao JQ, Fan J, Li Y, He P, Xiao SY, Wang WM. Multiple intramolecular trafficking signals in RESISTANCE TO POWDERY MILDEW 8.2 are engaged in activation of cell death and defense. Plant J. 2019;98:55-70.

48. Fu QT, Yu DQ. Expression profiles of AtWRKY25, AtWRKY26 and AtWRKY33 under abiotic stresses. Hereditas. 2010;32:848-56.

49. Babitha KC, Ramu SV, Pruthvi V, Mahesh P, Karaba NN, Madan U. Coexpression of AtbHLH17 and AtWRKY28 confers resistance to abiotic stress in Arabidopsis. Transgenic Res. 2013;22(2):327-41.

50. Zhao H, Wang S, Chen S, Jiang J, Liu GF. Phylogenetic and stress-responsive expression analysis of 20 WRKY genes in Populus simonii $\times$ Populus nigra. Gene. 2015;565:130-9.

51. Karanja BK, Fan L, Xu L, Wang Y, Zhu XW, Tang MJ, Wang RH, Zhang F, Muleke EM, Liu LW. Genome-wide characterization of the WRKY gene family in radish (Raphanus sativus $L$.) reveals its critical functions under different abiotic stresses. Plant Cell Rep. 2017;36:1757-73.

52. Ishiguro S, Nakamura K. Characterization of a cDNA encoding a novel DNA. Binding protein, SPF1, that recognizes SP8 sequences in the $5^{\prime}$ upstream regions of genes coding for sporamin and pamylase from sweet potato. Mol Gen Genet. 1994;244:563-71.

53. Kumar R, Tyagi AK, Sharma AK. Genome-wide analysis of auxin response factor (ARF) gene family from tomato and analysis of their role in flower and fruit development. Mol Gen Genomics. 2011;285(3):245-60.

54. Ulker B, Somssich IE. WRKY transcription factors: from DNA binding towards biological function. Curr Opin Plant Biol. 2004;7:491-8.

55. Liu JJ, Ekramoddoullah AK. Identification and characterization of the WRKY transcription factor family in Pinus monticola. Genome. 2009;52:77-88.

56. Rushton PJ, Somssich IE, Ringler P, Shen QJ. WRKY transcription factors. Trends Plant Sci. 2010;15:247-58.

57. Schauser L, Wieloch W, Stougaard J. Evolution of NIN-like proteins in Arabidopsis, rice, and Lotus japonicus. J Mol Evol. 2005;60(2):229-37.
58. Cannon S, Mitra A, Baumgarten A, Young N, May G. The roles of segmental and tandem gene duplication in the evolution of large gene families in Arabidopsis thaliana. BMC Plant Biol. 2004;4:10.

59. Diao WP, Snyder JC, Wang SB, Liu JB, Pan BG, Guo GJ, Wei G. Genome-wide identification and expression analysis of WRKY gene family in Capsicum annuum L. Front Plant Sci. 2016;7.

60. Wu KL, Guo ZJ, Wang HH. The WRKY family of transcription factors in rice and Arabidopsis and their origins. DNA Res. 2005;12:9-26.

61. Jiang SY, González JM, Srinivasan R. Comparative genomic and transcriptomic analysis of tandemly and segmentally duplicated genes in rice. PLoS One. 2013;8(5):e63551.

62. Huang SX, Gao YF, Liu JK. Genome wide analysis of WRKY transcription factors in Solanumly copersicum. Mol Gen Genomics. 2012;287:495-513.

63. Ling $L$, Jiang WJ, Zhang Y. Genome-wide analysis of WRKY gene family in Cucumis sativus L. BMC Genomics. 2011:12:471.

64. Celik Altunoglu Y, Baloglu P, Yer EN, Pekol S, Baloglu MC. Identification and expression analysis of LEA gene family members in cucumber genome. Plant Growth Regul. 2016;80(2):225-41.

65. Yu J, Wang L, Guo H, Liao B, King G, Zhang X. Genome evolutionary dynamics followed by diversifying selection explains the complexity of the Sesamum indicum genome. BMC Genomics. 2017;18:257.

66. Li DH, Liu P, Yu JY, Wang LH, Dossa K, Zhang YX, Zhou R, Wei X. Genomewide analysis of wrky gene family in the sesame genome and identification of the WRKY genes involved in responses to abiotic stresses. BMC Plant Biol. 2017;17(1):152.

67. Wei KF, Chen J, Chen YF, Wu LJ, Xie DX. Molecular phylogenetic and expression analysis of the complete WRKY transcription factor family in maize. DNA Res. 2012;19(2):153-64

68. Zhang $Y$, Wang $L$. The WRKY transcription factor superfamily: its origin in eukaryotes and expansion in plants. BMC Evol Biol. 2005;5:1-12.

69. Niu CF, Wei W, Zhou QY, Tian AG, Hao YJ, Zhang WK, Ma B, Lin Q, Zhang ZB, Zhang JS, Chen SY. Wheat WRKY genes TaWRKY2 and TaWRKY19 regulate abiotic stress tolerance in transgenic Arabidopsis plants. Plant Cell Environ. 2012;35:1156-70.

70. Wang $X$, Zeng J, Li Y, Rong X, Sun J, Sun T, Li M, Wang L, Feng Y, Chai R, Chen M, Chang J, Li K, Yang G, He G. Expression of TaWRKY44, a wheat WRKY gene, in transgenic tobacco confers multiple abiotic stress tolerances. Front Plant Sci. 2015;6:615.

71. Wu XL, Shiroto Y, Kishitani S, Ito Y, Toriyama K. Enhanced heat and drought tolerance in transgenic rice seedlings overexpressing OsWRKY11 under the control of HSP101 promoter. Plant Cell Rep. 2009;28:21-30.

72. Wang F, Chen HW, Li QT, Wei W, Li W, Zhang WK, Ma B, Bi YD, La YC, Liu XL, Man WQ, Zhang JS, Chen SY. GmWRKY27 interacts with GmMYB174 to reduce expression of GmNAC29 for stress tolerance in soybean plants. Plant J. 2015;83:224-36

73. Wang F, Hou XL, Tang J, Wang Z, Wang SM, Jiang FL, Li Y. A novel coldinducible gene from Pak-choi (Brassica campestris ssp. chinensis), BcWRKY46, enhances the cold, salt and dehydration stress tolerance in transgenic tobacco. Mol Biol Rep. 2012;239:4553-64.

74. Liu QL, Zhong M, Li S, Pan YZ, Jiang BB, Jia Y, Zhang HQ. Overexpression of a chrysanthemum transcription factor gene, DgWRKY3, in tobacco enhances tolerance to salt stress. Plant Physiol Biochem. 2013;69:27-33.

75. Jia HH, Wang C, Wang F, Liu SC, Li GL, Guo XQ. GhWRKY68 reduces resistance to salt and drought in transgenic Nicotiana benthamiana. PLoS ONE. 2015;10:e0120646.

76. Krzywinski M, Schein J, Birol I, Connors J, Gascoyne R, Horsman D. Circos: an information aesthetic for comparative genomics. Genome Res. 2009;19(9): 1639-45.

77. Kumar S, Stecher G, Tamura K. MEGA7: molecular evolutionary genetics analysis version 7.0 for bigger datasets. Mol Biol Evol. 2016;33(7):msw054.

78. Lu Y, Sun J, Yang ZM, Zhao CX, Zhu MK, Ma DF, Dong TT, Zhou ZK, Liu MY, Yang DJ, Li ZY, Xu T. Genome-wide identification and expression analysis of glycine-rich RNA-binding protein family in sweet potato wild relative Ipomoea trifida. Gene. 2019;686:177-86.

\section{Publisher's Note}

Springer Nature remains neutral with regard to jurisdictional claims in published maps and institutional affiliations. 